\title{
Hyperacute drug-induced hepatitis with intravenous amiodarone: case report and review of the literature
}

This article was published in the following Dove Press journal:

Drug, Healthcare and Patient Safety

25 September 2013

Number of times this article has been viewed

\section{Mohammad Nasser \\ Timothy R Larsen \\ Barryton Waanbah \\ Ibrahim Sidiqi \\ Peter A McCullough}

Providence Hospitals and Medical Centers, Department of Medicine, Division of Cardiology, Southfield and Novi, MI, USA
Correspondence: Mohammad Nasser Internal Medicine, Providence Hospitals and Medical Centers, I600I West Nine Mile Rd, Southfield, MI 48075, USA Email mnasser23@yahoo.com

\begin{abstract}
Amiodarone is a benzofuran class III antiarrhythmic drug used to treat a wide spectrum of ventricular tachyarrhythmias. The parenteral formulation is prepared in polysorbate 80 diluent. We report an unusual case of acute elevation of aminotransaminase concentrations after the initiation of intravenous amiodarone. An 88-year-old Caucasian female developed acute hepatitis and renal failure after initiating intravenous amiodarone for atrial fibrillation with a rapid ventricular response in the setting of acutely decompensated heart failure and hepatic congestion. Liver transaminases returned to baseline within 7 days after discontinuing the drug. Researchers hypothesized that this type of injury is related to liver ischemia with possible superimposed direct drug toxicity. The CIOMS/RUCAM scale identifies our patient's acute hepatitis as a highly probable adverse drug reaction. Future research is needed to understand the mechanisms by which hyperacute drug toxicity occurs in the setting of impaired hepatic perfusion and venous congestion.
\end{abstract}

Keywords: intravenous amiodarone, acute hepatotoxicity, liver transaminases, drug-induced liver toxicity

\section{Introduction}

Amiodarone is a benzofuran class III antiarrhythmic drug used to treat a wide spectrum of ventricular and supraventricular tachyarrhythmias. The mechanism of action is complex. It involves antagonism of the delayed rectifier potassium channels, in particular the rapid component, thereby increasing membrane refractoriness. This agent also affects inactivated sodium channels (Phase 0), sympathetic activity, and calcium channels (L-type). ${ }^{1}$ Long-term therapy is associated with various adverse effects due to accumulation of the drug in tissue. The intravenous (IV) preparation of amiodarone has been linked to adverse hepatic reactions such as hypotension, cardiac arrest, bradycardia, heart failure, and hepatic abnormalities. ${ }^{2}$ We report an unusual case of acute hepatitis immediately after the initiation of IV amiodarone.

\section{Case description}

An 88-year-old Caucasian female presented to the emergency room complaining of shortness of breath with minimal exertion. This was associated with intermittent heart palpitations and fatigue. Her symptoms had gradually worsened over the prior month in response to family and emotional stress. She denied chest pain, cough, hemoptysis, nausea, vomiting, or diarrhea.

Her past medical history consisted of hypothyroidism, hypertension, and depression. Her only prior surgery was a hysterectomy. A recent echocardiogram 
revealed a normal ejection fraction with Grade 1 diastolic dysfunction. Family history was unremarkable. She did not use tobacco, alcohol, or illicit drugs. Her home medications included sertraline $25 \mathrm{mg}$ daily, levothyroxine $25 \mathrm{mg}$ daily, and lisinopril $20 \mathrm{mg}$ daily.

A physical examination revealed the following vitals: a blood pressure of $147 / 52 \mathrm{mmHg}$, a temperature of $97.6^{\circ} \mathrm{F}$, a respiratory rate of 20 breaths/minute, and a heart rate of 130 beats/minute. Cardiac auscultation demonstrated an irregular rhythm with a diastolic murmur heard best at the left upper sternal border, likely to be aortic in origin. An S3 gallop was present, and point of maximal impulse was laterally displaced. Auscultation of the lungs revealed bibasilar rales. Peripheral pulses were strong and equal bilaterally. There was moderate edema present in the lower extremities, and hepatojugular reflux was noted.

Laboratory testing identified the following values: white blood cell count of $8.8 \times 10^{3} / \mu \mathrm{L}$, hemoglobin of $13.1 \mathrm{~g} / \mathrm{dL}$, platelet count of $277 \times 10^{3} / \mu \mathrm{L}$, glucose level of $108 \mathrm{mg} / \mathrm{dL}$, creatinine of $61.88 \mu \mathrm{mol} / \mathrm{L}$, aspartate aminotransferase (AST) of 24 units/L, alanine aminotransferase (ALT) of 16 units/L, bilirubin total of $8.5 \mu \mathrm{mol} / \mathrm{L}$, alkaline phosphatase of 98 units/L, and thyroid-stimulating hormone of $2.92 \mu \mathrm{IU} / \mathrm{mL}$. All electrolytes were within normal limits. An initial electrocardiogram (ECG) revealed atrial fibrillation with a rapid ventricular response (Figure 1).

Intravenous diltiazem was initiated in order to control the ventricular rate. Shortly after, the patient's rhythm converted to normal sinus rhythm spontaneously. She subsequently developed sinus pauses lasting up to 6 seconds; consequently, diltiazem was discontinued. Until a permanent pacemaker could be inserted, IV amiodarone was commenced in order to maintain sinus rhythm and prevent a rapid ventricular response. Following a loading dose of $150 \mathrm{mg}$, we administered $360 \mathrm{mg}$ of amiodarone infused at a rate of $1 \mathrm{mg} / \mathrm{min}$ over 6 hours, after which a maintenance infusion rate of $0.5 \mathrm{mg} / \mathrm{min}$ was continued.

The next day, a routine laboratory evaluation illustrated an acute elevation to the following measurements: AST 1,881 units/L (normal high 35 units/L), ALT 1,048 units/L (normal high 35 units/L), alkaline phosphatase 143 units/L (normal high 129 units/L), total bilirubin $15.3 \mu \mathrm{mol} / \mathrm{L}$ (normal high $17 \mu \mathrm{mol} / \mathrm{L}$ ), and creatinine $97.2 \mu \mathrm{mol} / \mathrm{L}$ (normal high $88 \mu \mathrm{mol} / \mathrm{L}$ ) (Table 1$)$. At that point, we reviewed all medications and obtained a hepatitis panel, which was normal. She had been on the same home medications for months without any change. A hepatic ultrasound identified venous congestion. We suspected amiodarone as a cause; thus, it was discontinued after administering a total dose of $960 \mathrm{mg}$ over a 10-hour period. Signs of a hypersensitivity reaction such as itching, rash, or eosinophilia were not seen. Liver transaminases returned to baseline within 7 days. Further investigation with a cardiac echocardiogram demonstrated a left ventricular ejection fraction (LVEF) of 35\%. Subsequently, a left heart catheterization revealed significant coronary artery disease with no clear revascularization targets, and a LVEF of $30 \%$. She then received a permanent pacemaker, made an uneventful recovery, and was discharged on carvedilol, lisinopril, warfarin, and levothyroxine. Over the next 12 weeks, the patient suffered from progressive heart failure, which was

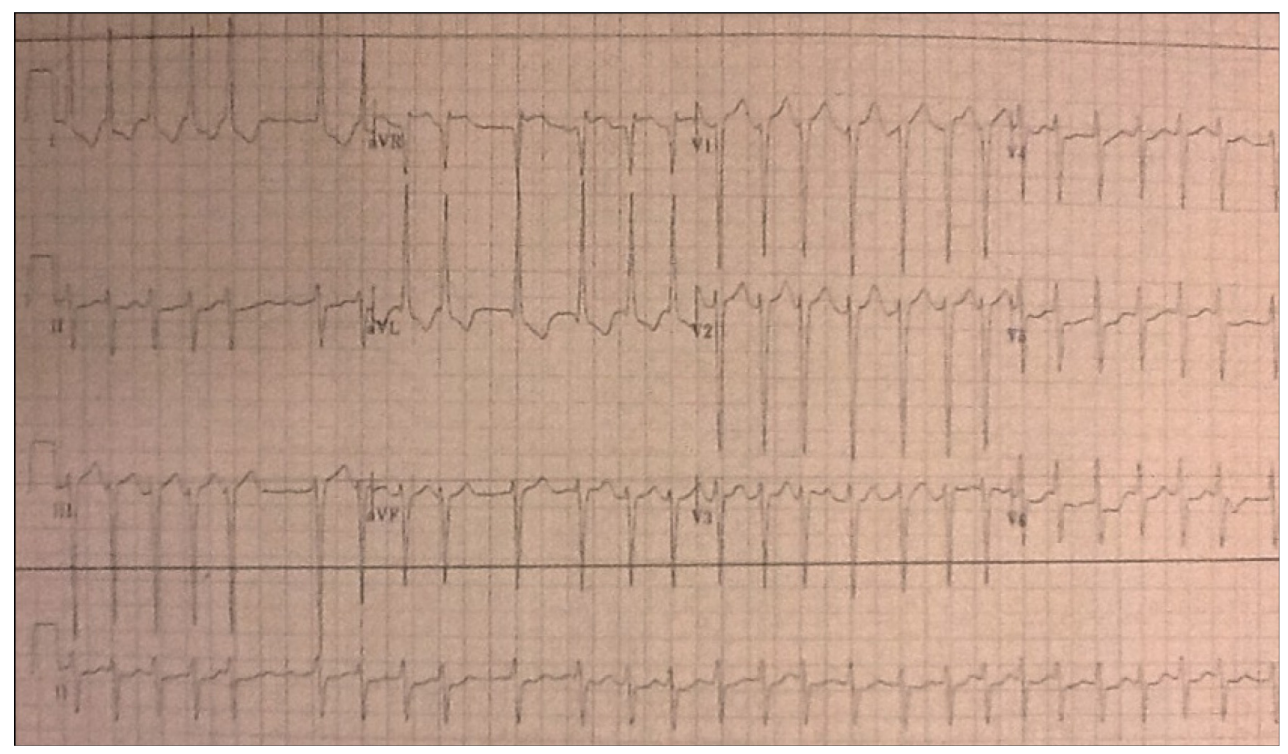

Figure I Standard 12-lead electrocardiogram demonstrating atrial fibrillation with rapid ventricular response. 
Table I Liver-enzyme measurements

\begin{tabular}{llll}
\hline Day of hospitalization & $\begin{array}{l}\text { AST } \\
(\mathbf{U} / \mathbf{L})\end{array}$ & $\begin{array}{l}\text { ALT } \\
(\mathbf{U} / \mathbf{L})\end{array}$ & $\begin{array}{l}\text { Alk Phos } \\
\text { (U/L) }\end{array}$ \\
\hline $\begin{array}{l}\text { Day I } \\
\quad \text { Amio started }\end{array}$ & 24 & 16 & 98 \\
$\begin{array}{l}\text { Day 2 } \\
\text { Amio discontinued }\end{array}$ & $1,88 \mathrm{I}$ & 1,048 & 143 \\
Day 3 & & & \\
Day 4 & 613 & 678 & 155 \\
Day 5 & 328 & 578 & 174 \\
Day 6 & 221 & 470 & 160 \\
\hline
\end{tabular}

Abbreviations: Alk Phos, alkaline phosphatase; ALT, alanine aminotransferase Amio, amiodarone; AST, aspartate aminotransferase.

managed both in the office and at home. Eventually, she died of advanced pump failure with progressive edema and respiratory failure.

\section{Discussion}

Major side effects of oral amiodarone are related to drug accumulation in tissue when given over a long period of time. Adverse reactions include thyroid dysfunction, sinus bradycardia, ventricular arrhythmias, and pulmonary and hepatic toxicity. Approximately $25 \%$ of patients taking this medication develop a transient asymptomatic rise in serum aminotransferase levels. Symptomatic hepatitis, cirrhosis, and hepatic failure are rare complications which involve less than $3 \%$ of patients. ${ }^{3,4}$ Histological features of oral amiodarone hepatitis are similar to alcoholic hepatitis and include steatosis, fibrosis, and phospholipid laden lysosomal lamellar bodies. The CIOMS/RUCAM scale identifies our patient's acute hepatitis as a highly probable adverse drug reaction. ${ }^{5}$ There was a mild total bilirubin elevation to $0.9 \mathrm{mg} / \mathrm{dL}$; therefore, this case of drug-induced liver injury (DILI) did not meet Hy's Law criteria, which states that hepatocellular injury accompanied by a total bilirubin elevation over twice the upper limit of normal is of significant concern and has a mortality of $10 \%-15 \% .^{6}$

Intravenous amiodarone is typically used as a short-term therapy for various arrhythmias (as mentioned previously). It is metabolized to N-desethylamiodarone (DEA) by cytochrome P450 enzymes (CYP3A4 and CYP2C8). Its metabolite is also an antiarrhythmic. Amiodarone is primarily eliminated by biliary excretion. Left ventricular dysfunction prolongs the half-life of DEA. Acute hepatitis, due to parenteral therapy, is extremely rare. ${ }^{7}$ Our literature review identified 33 previously reported cases. The underlying mechanism is controversial and still unknown. Ischemic hepatitis, a much more common condition, shares many clinical and histological characteristics that are seen in parenteral amiodarone-induced liver injury. It has been hypothesized by Gluck et al that the acute liver injury following the IV formulation is related to liver ischemia, rather than direct drug toxicity. ${ }^{8}$ This was based on the observation that the two conditions show similar histological features and clinical events. Furthermore, DILI caused by oral and IV amiodarone

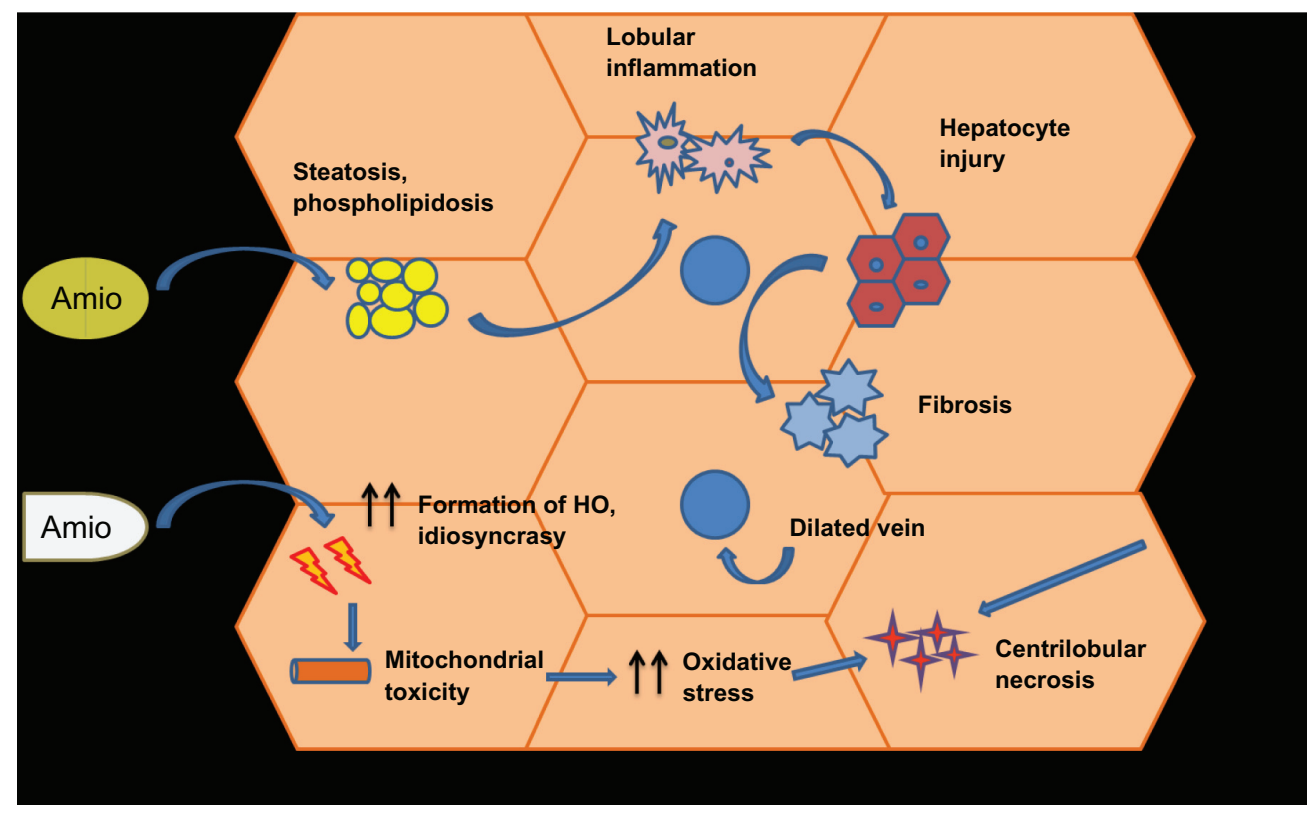

Figure 2 Pattern of hepatocyte injury with oral amiodarone, intravenous amiodarone, and hepatic hypoperfusion.

Notes: Intravenous amiodarone is thought to have direct cell toxicity with free radical formation and impairment of mitochondrial function, which leads to centrilobular necrosis.

Abbreviation: Amio, amiodarone; HO, hydroxyl radical. 
Table 2 Published cases of fatal intravenous amiodarone-induced acute hepatitis

\begin{tabular}{|c|c|c|c|c|c|c|}
\hline Year & Authors & Age & Sex & Indication & Associated conditions & $\begin{array}{l}\text { AST/ALT } \\
(\times U L N)\end{array}$ \\
\hline \multirow[t]{2}{*}{1986} & Lupon-Roses et al" & 77 & M & Atrial tachycardia & Jaundice & AST: 47 \\
\hline & & & & & & ALT: 29 \\
\hline \multirow[t]{2}{*}{1988} & Pye et al ${ }^{12}$ & 48 & $\mathrm{~F}$ & Atrial fibrillation & MR (moderate) & AST: 47 \\
\hline & & & & & & ALT: 32 \\
\hline \multirow[t]{2}{*}{1988} & Pye et $\mathrm{al}^{12}$ & 70 & $\mathrm{~F}$ & Atrial fibrillation & $\mathrm{HF}, \mathrm{MI}, \mathrm{MR}, \mathrm{PH}$ & AST: 60 \\
\hline & & & & & & ALT: 26 \\
\hline 1989 & Stevenson et $\mathrm{al}^{13}$ & 59 & M & Atrial fibrillation & HF, hepatomegaly, jaundice & AST: 10 \\
\hline \multirow[t]{2}{*}{1990} & Simon et $\mathrm{al}^{14}$ & 59 & M & Atrial fibrillation & Pulmonary edema & AST: 37 \\
\hline & & & & & & ALT: 38 \\
\hline \multirow[t]{2}{*}{1991} & Morelli et al ${ }^{15}$ & 58 & M & Atrial fibrillation & Jaundice, nausea & AST: I5 \\
\hline & & & & & & ALT: 5 \\
\hline \multirow[t]{2}{*}{1991} & Morelli et $\mathrm{al}^{15}$ & 68 & M & Ventricular fibrillation & Hepatomegaly, edema, HF & AST: 4 \\
\hline & & & & & & ALT: 2 \\
\hline \multirow[t]{2}{*}{ 1991 } & Kalantzis et al ${ }^{16}$ & 28 & M & Atrial fibrillation & Jaundice, hepatomegaly, renal & AST: 501 \\
\hline & & & & & failure & ALT: 370 \\
\hline \multirow[t]{2}{*}{1991} & Kalantzis et $\mathrm{a}^{16}$ & 60 & M & Atrial fibrillation & Hepatomegaly, renal failure & AST: 30 \\
\hline & & & & & & ALT: 10 \\
\hline \multirow[t]{2}{*}{1992} & Fornaciari et $\mathrm{al}^{17}$ & 52 & $\mathrm{~F}$ & Ventricular tachycardia & Hepatomegaly & AST: 50 \\
\hline & & & & & & ALT: 45 \\
\hline \multirow[t]{2}{*}{1993} & Rhodes et $\mathrm{al}^{18}$ & 72 & M & Ventricular tachycardia & Oliguria, hepatic encephalopathy & AST: |3| \\
\hline & & & & & & ALT: 132 \\
\hline \multirow[t]{2}{*}{1995} & Tosetti et al ${ }^{19}$ & 66 & M & Atrial fibrillation & Nausea, oliguria & AST: 9 \\
\hline & & & & & & ALT: II \\
\hline \multirow[t]{2}{*}{1996} & Paniagua et $\mathrm{a}^{20}$ & 80 & $\mathrm{~F}$ & Atrial fibrillation & Hepatomegaly & AST: 82 \\
\hline & & & & & & ALT: 59 \\
\hline 1997 & James et $\mathrm{al}^{21}$ & 50 & M & Atrial fibrillation & Dyspnea & AST: 205 \\
\hline \multirow[t]{2}{*}{1997} & Tagliamonte et $\mathrm{al}^{22}$ & 61 & M & Ventricular tachycardia & Jaundice & AST: 243 \\
\hline & & & & & & ALT: 122 \\
\hline \multirow[t]{2}{*}{1998} & Breuer et $\mathrm{a}^{23}$ & 64 & M & Atrial fibrillation & HF, renal failure, anemia, & AST: 63 \\
\hline & & & & & hypotension & ALT: 69 \\
\hline \multirow[t]{2}{*}{1999} & Iliopoulou et $\mathrm{a}^{24}$ & 69 & M & Premature ventricular & Angina & AST: 50 \\
\hline & & & & complexes & & ALT: 50 \\
\hline 1999 & Lopez-Gamez et $\mathrm{al}^{25}$ & 60 & M & Ventricular tachycardia & Jaundice & ALT: 57 \\
\hline \multirow[t]{2}{*}{2000} & Luengo et $\mathrm{al}^{26}$ & 68 & $\mathrm{~F}$ & Atrial fibrillation & Hepatomegaly & AST: 14 \\
\hline & & & & & & ALT: 37 \\
\hline \multirow[t]{2}{*}{2002} & Gregory et $\mathrm{a}^{28}$ & 74 & $\mathrm{~F}$ & Ventricular tachycardia & Dyspnea & AST: 13 \\
\hline & & & & & & ALT: 8 \\
\hline \multirow[t]{2}{*}{2002} & Gonzalez et $\mathrm{a}^{27}$ & 69 & $\mathrm{~F}$ & Atrial fibrillation & Jaundice & AST: 195 \\
\hline & & & & & & ALT: 227 \\
\hline \multirow[t]{2}{*}{2002} & Agozzino et $\mathrm{a}^{29}$ & 83 & $\mathrm{~F}$ & Atrial fibrillation & $\mathrm{HF}$, oliguria & AST: 365 \\
\hline & & & & & & ALT: 135 \\
\hline 2002 & Giannattasio et $\mathrm{al}^{30}$ & 65 & M & Supraventricular tachycardia & Jaundice, hepatomegaly, edema & ALT: 100 \\
\hline 2002 & Giannattasio et $\mathrm{al}^{30}$ & 55 & M & Atrial fibrillation & Core pulmonaly, hepatomegaly & ALT: 10 \\
\hline 2002 & Giannattasio et $\mathrm{al}^{30}$ & 75 & $\mathrm{~F}$ & Supraventricular tachycardia & Jaundice, ascites & ALT: 60 \\
\hline \multirow[t]{2}{*}{2005} & Rätz Bravo et $\mathrm{al}^{31}$ & 66 & $\mathrm{~F}$ & Atrial fibrillation & HF, postop & AST: 106 \\
\hline & & & & & & ALT: 66 \\
\hline 2005 & Rätz Bravo et $\mathrm{a}^{31}$ & 73 & $\mathrm{~F}$ & Atrial fibrillation & Postop, no hypotension & AST: 485 \\
\hline & & & & & & ALT: 206 \\
\hline 2005 & Rätz Bravo et $\mathrm{al}^{31}$ & 57 & M & Atrial fibrillation & HF, post CABG, low MAP & AST: 44 \\
\hline & & & & & during surgery & ALT: 5 I \\
\hline
\end{tabular}




\begin{tabular}{|c|c|c|c|c|c|}
\hline $\begin{array}{l}\text { ALK PHOS/BILI } \\
(\times U L N)\end{array}$ & $\begin{array}{l}\text { Creatinine } \\
(\mu \mathrm{mol} / \mathrm{L})\end{array}$ & $\begin{array}{l}\text { Latency } \\
\text { period }\end{array}$ & $\begin{array}{l}\text { Cumulative } \\
\text { amio dose (mg) }\end{array}$ & $\begin{array}{l}\text { Oral rechallenge } \\
\text { test }\end{array}$ & Fatality \\
\hline BILI: 5 & - & 3 days & 2,300 & - & - \\
\hline BILI: 7 & - & I day & 1,200 & Negative test & - \\
\hline BILI: 3 & - & 7 days & 8,700 & - & - \\
\hline $\begin{array}{l}\text { ALK PHOS: } 2 \\
\text { BILI: } 17\end{array}$ & - & I day & 450 & - & - \\
\hline BILI: Nrml & - & I day & 1,200 & $\begin{array}{l}\text { Positive test with } \\
\text { intravenous amiodarone }\end{array}$ & - \\
\hline $\begin{array}{l}\text { ALK PHOS: } 5 \\
\text { BILI: } 3\end{array}$ & - & 3 days & 3,070 & Negative test & - \\
\hline $\begin{array}{l}\text { ALK PHOS: } 5 \\
\text { BILI: } 3\end{array}$ & - & 6 days & 2,375 & Negative test & - \\
\hline BILI: 6 & $\begin{array}{l}\text { 963-required } \\
\text { HD }\end{array}$ & I day & $\mathrm{I}, 500$ & - & $\begin{array}{l}\text { Died after I } 4 \text { days of } \\
\text { hepatorenal failure and } \\
\text { coma }\end{array}$ \\
\hline BILI: 5 & 274 & I day & $\mathrm{I}, 500$ & - & $\begin{array}{l}\text { Died after } 4 \text { days of } \\
\text { hepatic coma and } \\
\text { renal failure }\end{array}$ \\
\hline BILI: 29 & - & 36 hours & 1,200 & - & - \\
\hline BILI: 3 & 328 & 12 hours & $\mathrm{I}, 200$ & Negative test & - \\
\hline ALK PHOS: I.3 & - & I day & 400 & - & - \\
\hline- & 254 & I day & 750 & - & - \\
\hline- & - & I day & 1,200 & Negative test & - \\
\hline ALK PHOS: 2.5 & - & I day & - & - & - \\
\hline BILI: 10 & & & & & \\
\hline- & - & 4 days & 3,400 & - & - \\
\hline BILI: increased & - & I day & 1,500 & - & - \\
\hline BILI: 8 & - & 7 days & 2,400 & Negative test & - \\
\hline- & - & 5 days & 1,300 & - & - \\
\hline- & - & I day & $\mathrm{I}, 740$ & Negative test & - \\
\hline BILI: 4 & - & I day & 1,200 & - & - \\
\hline BILI: 19 & 300 & I day & 1,000 & - & - \\
\hline BILI: 4 & - & I day & 600 & - & - \\
\hline- & - & 3 days & 600 & Negative test & \\
\hline BILI: 19 & - & I day & 600 & - & $\begin{array}{l}\text { Died after } 3 \text { I days of } \\
\text { hepatic failure }\end{array}$ \\
\hline $\begin{array}{l}\text { ALK PHOS: Nrml } \\
\text { BILI: Nrml }\end{array}$ & - & I day & 200 & - & - \\
\hline $\begin{array}{l}\text { ALK PHOS: } 2 \\
\text { BILI: Nrml }\end{array}$ & - & 14 hours & 720 & - & - \\
\hline $\begin{array}{l}\text { ALK PHOS; Nrml } \\
\text { BILI: } 2\end{array}$ & - & I day & 890 & - & - \\
\hline
\end{tabular}


Table 2 (Continued)

\begin{tabular}{|c|c|c|c|c|c|c|}
\hline Year & Authors & Age & Sex & Indication & Associated conditions & $\begin{array}{l}\text { AST/ALT } \\
\text { (xULN) }\end{array}$ \\
\hline \multirow[t]{2}{*}{2008} & Chan et $\mathrm{al}^{34}$ & 72 & $\mathrm{~F}$ & Atrial fibrillation & Hypotension, jaundice, oliguria & AST: 33 \\
\hline & & & & & & ALT: 42 \\
\hline \multirow[t]{2}{*}{2008} & Cataldi et $\mathrm{al}^{32}$ & 77 & $\mathrm{~F}$ & Atrial fibrillation & HF, fluid overload & AST: 53 \\
\hline & & & & & & ALT: 40 \\
\hline \multirow[t]{2}{*}{2009} & Murphy et al ${ }^{10}$ & 59 & $M$ & Atrial fibrillation & LVH & AST: 172 \\
\hline & & & & & & ALT: 83 \\
\hline 2012 & Lahbabi et al $^{9}$ & 29 & $\mathrm{~F}$ & Atrial fibrillation & Severe MR, LV dilation & ALT: 19 \\
\hline 2012 & Grecian et $\mathrm{al}^{33}$ & 73 & $M$ & Ventricular tachycardia & Hx of HF, fulminant hepatic failure & ALT: 44 \\
\hline
\end{tabular}

Abbreviations: ALK, alkaline; PHOS, phosphatase; BILI, bilirubin total; AST, aspartate aminotransferase; ALT, alanine aminotransferase; HF, heart failure; MR, mitral regurgitation; PH, pulmonary hypertension; MAP, mean arterial pressure; CABG, coronary artery bypass grafting; MI, myocardial infarction; HD, hemodialysis; Nrml, normal; Amio, amiodarone; LVH, left ventricular hypertrophy; ULN, upper limit of normal; Hx, history; LV, left ventricle.

demonstrate different histologic findings. The majority of patients receiving the IV form are suffering from unstable tachyarrhythmias, which may result in a decreased cardiac output, hypotension, and ischemia. Most patients described in the reviewed cases had evidence of poor forward output, hepatic venous congestion, impaired circulation and acute kidney injury, predisposing them to ischemic hepatitis. Finally, another report by Lahbabi et al ascribes responsibility of liver toxicity to solubilizers such as polysorbate 80 in the IV amiodarone preparation. ${ }^{9}$ Polysorbate 80 has been implicated in the E-Ferol syndrome characterized by renal failure, hepatosplenomegaly, and jaundice. Eliminating polysorbate 80 by the oral route demonstrated the safe use of amiodarone even after acute hepatitis in several studies.

Our patient showed evidence of impaired left ventricular function with an LVEF of $30 \%$ by ECG (decreased from $50 \%$ one month prior to admission). Also, there was evidence of acute elevation of her creatinine from 0.7 to $1.1 \mathrm{mg} / \mathrm{dL}$, suggestive of a degree of hypoperfusion. Hepatojugular reflux was elicited and central venous pressure was elevated

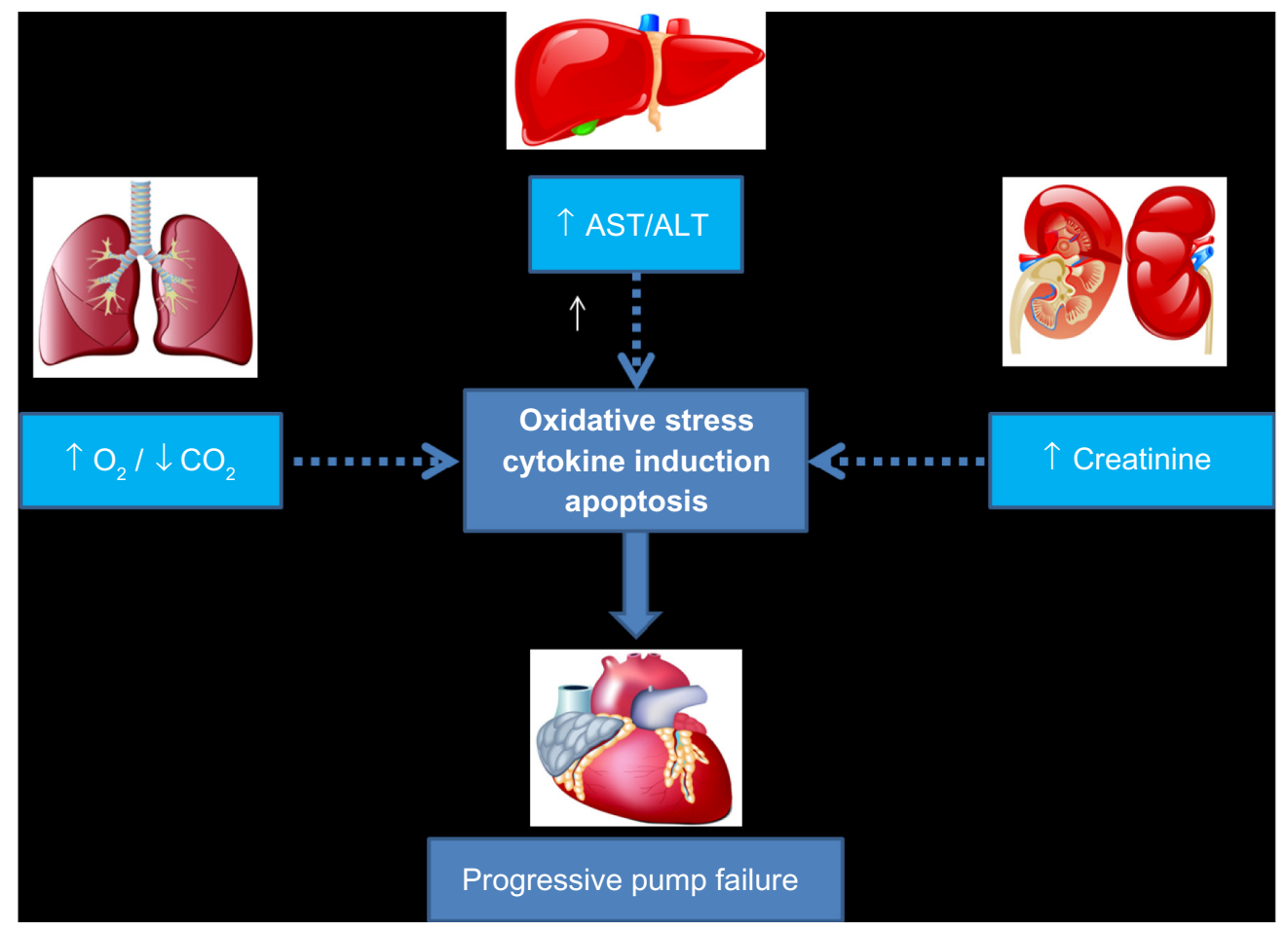

Figure 3 Schematic representation of multiorgan failure contributing to systemic inflammation, oxidative stress, and apoptosis, which contributes to progressive pump failure and death.

Abbreviations: ALT, alanine aminotransferase; AST, aspartate aminotransferase; $\mathrm{O}_{2}$, Oxygen; $\mathrm{CO}_{2}$, carbon dioxide. 


\begin{tabular}{|c|c|c|c|c|c|}
\hline $\begin{array}{l}\text { ALK PHOS/BILI } \\
(\times U L N)\end{array}$ & $\begin{array}{l}\text { Creatinine } \\
(\mu \mathrm{mol} / \mathrm{L})\end{array}$ & $\begin{array}{l}\text { Latency } \\
\text { period }\end{array}$ & $\begin{array}{l}\text { Cumulative } \\
\text { amio dose (mg) }\end{array}$ & $\begin{array}{l}\text { Oral rechallenge } \\
\text { test }\end{array}$ & Fatality \\
\hline $\begin{array}{l}\text { ALK PHOS: I.5 } \\
\text { BILI: I.I }\end{array}$ & 274 & I day & 2,550 & - & $\begin{array}{l}\text { Died after } 20 \text { days } \\
\text { of hepatic coma and } \\
\text { renal failure }\end{array}$ \\
\hline BILI: 2 & - & I day & 750 & - & $\begin{array}{l}\text { Died after } 29 \text { days of } \\
\text { multiorgan failure }\end{array}$ \\
\hline $\begin{array}{l}\text { ALK PHOS: I.5 } \\
\text { BILI: } 68\end{array}$ & 236 & I day & 1,200 & - & $\begin{array}{l}\text { Died after } 4 \text { days of } \\
\text { acute hepatic failure }\end{array}$ \\
\hline $\begin{array}{l}\text { ALK PHOS: Nrml } \\
\text { BILI: Nrml }\end{array}$ & 70 & I day & $\mathrm{I}, 599$ & Negative test & - \\
\hline BILI: 5 & 274 & I day & 900 & - & - \\
\hline
\end{tabular}

subsequent to liver injury. The causal correlation is not clear as to which condition (cardio-renal dysfunction or liver failure) induced the other. We believe that in susceptible elderly patients, even the standard intravenous amiodarone dose may cause direct drug toxicity and hypotension, especially in the setting of heart failure, leading to hepatic injury.

Further research is needed to support a true acute amiodarone hepatotoxicity versus other proposed or even unknown mechanisms (Figure 2). Six fatal cases of IV amiodarone hepatitis have been reported, suggesting the severity of this condition (Table 2). ${ }^{10-34}$ Elevation of transaminases occurred within 24 hours of drug administration in most patients. The majority of these cases were associated with some degree of cardiac dysfunction and renal failure. Our case was fatal in 12 weeks due to progressive pump failure, and the DILI event may have been a harbinger of mortality. The mechanism of progressive left ventricular failure may have been secondary to the impact of systemic inflammation, neurohormonal stress, and microcirculatory dysfunction caused in part by the acute organ failure of the liver (Figure 3). We believed persistent hepatic venous congestion played a role and may have been a determinant. This case suggests that one should regularly obtain a liver function panel subsequent to parenteral amiodarone initiation and proceed with caution in the setting of heart failure and hepatic congestion.

\section{Conclusion}

Amiodarone is often used to treat life-threatening arrhythmias in the setting of acutely decompensated heart failure. In the presence of hepatic congestion, the IV preparation of amiodarone may cause acute liver injury, which can be a harbinger for a fatal outcome in the days to months after administration.
Future research is needed to understand the mechanisms by which hyperacute drug toxicity occurs in the setting of impaired hepatic perfusion and venous congestion.

\section{Disclosure}

The authors report no conflicts of interest in this work.

\section{References}

1. Smith TW, Cain ME. Class III antiarrhythmic drugs: Amiodarone, ibutilide and sotalol. In Zipes DP, Jalife J, editors. Cardiac electrophysiology: From cell to bedside. 5th ed. Philadelphia, WB Saunders; 2009: 932-941.

2. Goldschlager N, Epstein AE, Naccarelli GV, et al; Practice Guidelines Sub-committee, North American Society of Pacing and Electrophysiology (HRS). A practical guide for clinicians who treat patients with amiodarone: 2007. Heart Rhythm. 2007;4(9): $1250-1259$.

3. Lewis JH, Ranard RC, Caruso A, et al. Amiodarone hepatotoxicity: prevalence and clinicopathologic correlations among 104 patients. Hepatology. 1989;9(5):679-685.

4. Richer M, Robert S. Fatal hepatotoxicity following oral administration of amiodarone. Ann Pharmacother. 1995;29(6):582-586.

5. Andrade RJ, Robles M, Fernández-Castañer A, López-Ortega S, López-Vega MC, Lucena MI. Assessment of drug-induced hepatotoxicity in clinical practice: a challenge for gastroenterologists. World $J$ Gastroenterol. 2007;13(3):329-340.

6. Reuben A. Hy's law. Hepatology. 2004;39:574-578.

7. Desai AD, Chun S, Sung RJ. The role of intravenous amiodarone in the management of cardiac arrhythmias. Ann Intern Med. 1997;127(4): 294-303.

8. Gluck N, Fried M, Porat R. Acute amiodarone liver toxicity likely due to ischemic hepatitis. Isr Med Assoc J. 2011;13(12):748-752.

9. Lahbabi M, Aqodad N, Ibrahimi A, Lahlou M, Aqodad H. Acute hepatitis secondary to parenteral amiodarone does not preclude subsequent oral therapy. World J Hepatol. 2012;4(6):196-198.

10. Murphy BP, Coldeway J, Raeside DA. Fatal acute fulminant hepatic failure caused by parenteral amiodarone: A case report and review of the literature. Scott Med J. 2009;54(1):58.

11. Lupon-Rosés J, Simó-Canonge R, Lu-Cortez L, Permanyer-Miralda G, Allende-Monclús H. Probable early acute hepatitis with parenteral amiodarone. Clin Cardiol. 1986;9(5):223-225.

12. Pye M, Northcote RJ, Cobbe SM. Acute hepatitis after parenteral amiodarone administration. Br Heart J. 1988;59(6): 690-691. 
13. Stevenson RN, Nayani TH, Davies JR. Acute hepatic dysfunction following parenteral amiodarone administration. Postgrad Med J. 1989;65(767):707-708.

14. Simon JP, Zannad F, Trechot P, Thisse JY, Houplon M, Aliot E. Acute hepatitis after a loading dose of intravenous amiodarone. Cardiovasc Drugs Ther. 1990;4(6):1467-1468.

15. Morelli S, Guido V, De Marzio P, Aguglia F, Balsano F. Early hepatitis during intravenous amiodarone administration. Cardiology. 1991; 78(3):291-294.

16. Kalantzis N, Gabriel P, Mouzas J, Tiniakos D, Tsigas D, Tiniakos G. Acute amiodarone-induced hepatitis. Hepatogastroenterology. 1991; 38(1):71-74.

17. Fornaciari G, Monducci I, Barone A, Bassi C, Beltrami M, Tomasi C. Amiodarone-induced acute hepatitis: case report. J Clin Gastroenterol. 1992;15(3):271-273.

18. Rhodes A, Eastwood JB, Smith SA. Early acute hepatitis with parenteral amiodarone: a toxic effect of the vehicle? Gut. 1993;34(4):565-566.

19. Tosetti C, Ongari M, Evangelisti A, Lolli R, Napoli A. Acute hepatotoxicity from amiodarone. Minerva Med. 1995;86(9):387-390. Italian.

20. Paniagua Clusells J, Arcusa Gavalda R, Goma Masip F, Pons Masanes S, Soler Masana JM. Acute hepatitis caused by intravenous amiodarone. Rev Esp Cardiol. 1996;49(5):384-385. Spanish.

21. James PR, Hardman SM. Acute hepatitis complicating parenteral amiodarone does not preclude subsequent oral therapy. Heart. 1997;77(6):583-584.

22. Tagliamonte E, Cice G, Ducceschi V, Mayer MS, Iacono A. Acute hepatitis following amiodarone administration. Minerva Cardioangiol. 1997;45(9):451-456. Italian.

23. Breuer HW, Bossek W, Haferland C, Schmidt M, Neumann H, Gruszka J. Amiodarone-induced severe hepatitis mediated by immunological mechanisms. Int J Clin Pharmacol Ther. 1998;36(6):350-352.

24. Iliopoulou A, Giannakopoulos G, Mayrikakis M, Zafiris E, Stamatelopoulos S. Reversible fulminant hepatitis following intravenous amiodarone loading. Amiodarone hepatotoxicity. Int $J$ Clin Pharmacol Ther. 1999;37(6):312-313.
25. López-Gómez D, Nicolás J, Frigola JM, Manito N, Esplugas E. The use of oral amiodarone as a chronic treatment in a patient with prior fulminant hepatitis due to intravenous amiodarone. Rev Esp Cardiol. 1999;52(3):201-203. Spanish.

26. Luengo O, Montero J, Alegre J, Fernández Sevilla T. Toxic hepatitis caused by intravenous amiodarone. Med Clin (Barc). 2000;115(20): 798-799. Spanish.

27. González Galilea A, García Sánchez MV, la Mata García M, Miño Fugarolas G. Early-onset acute toxic hepatitis induced by intravenous amiodarone administration. Gastroenterol Hepatol. 2002; 25(6):392-394. Spanish.

28. Gregory SA, Webster JB, Chapman GD. Acute hepatitis induced by parenteral amiodarone. Am J Med. 2002;113(3):254-255.

29. Agozzino F, Picca M, Pelosi G. Acute hepatitis complicating intravenous amiodarone treatment. Ital Heart J. 2002;3(11):686-688.

30. Giannattasio F, Salvio A, Varriale M, Picciotto FP, Di Costanzo GG, Visconti M. Three cases of severe acute hepatitis after parenteral administration of amiodarone: the active ingredient is not the only agent responsible for hepatotoxicity. Ann Ital Med Int. 2002;17(3): $180-184$.

31. Rätz Bravo AE, Drewe J, Schlienger RG, Krähenbühl S, Pargger H, Ummenhofer W. Hepatotoxicity during rapid intravenous loading with amiodarone: Description of three cases and review of the literature. Crit Care Med. 2005;33(1):128-134; discussion 245-246.

32. Cataldi A, Gonella D, Robutti N, Siri M, Buonocore S, Odetti P. Hepatotoxicity after intravenous amiodarone. Aging Clin Exp Res. 2008;20(6):593-596.

33. Grecian R, Ainslie M. Acute hepatic failure following intravenous amiodarone. BMJ Case Rep. 2012;doi:10.1136/bcr-2012-007080.

34. Chan AL, Hsieh HJ, Hsieh YA, Lin SJ. Fatal amiodarone-induced hepatotoxicity: a case report and literature review. Int J Clin Pharmacol Ther. 2008;46:96-101.
Drug, Healthcare and Patient Safety

\section{Publish your work in this journal}

Drug, Healthcare and Patient Safety is an international, peer-reviewed open-access journal exploring patient safety issues in the healthcare continuum from diagnostic and screening interventions through to treatment, drug therapy and surgery. The journal is characterized by the rapid reporting of reviews, original research, clinical, epidemiological and

\section{Dovepress}

post-marketing surveillance studies, risk management, health literacy and educational programs across all areas of healthcare delivery. The manuscript management system is completely online and includes a very quick and fair peer-review system. Visit http://www.dovepress.com/ testimonials.php to read real quotes from published authors. 